Communications in Mathematical Physics manuscript No.

(will be inserted by the editor)

\title{
Transformations on the set of all $n$-dimensional subspaces of a Hilbert space preserving principal angles
}

\author{
Lajos Molnár \\ Institute of Mathematics and Informatics, University of Debrecen, 4010 Debrecen, P.O.Box \\ 12, Hungary \\ E-mail: molnarl@math.klte.hu
}

November 19, 2018

To my wife for her unlimited(?) patience

\begin{abstract}
Wigner's classical theorem on symmetry transformations plays a fundamental role in quantum mechanics. It can be formulated, for example, in the following way: Every bijective transformation on the set $\mathcal{L}$ of all 1-dimensional subspaces of a Hilbert space $H$ which preserves the angle between the elements of $\mathcal{L}$ is induced by either a unitary or an antiunitary operator on $H$. The aim of this paper is to extend Wigner's result from the 1-dimensional case to the case of $n$-dimensional subspaces of $H$ with $n \in \mathbb{N}$ fixed.
\end{abstract}

\section{Introduction and statement of the main result}

Let $H$ be a (real or complex) Hilbert space and denote $B(H)$ the algebra of all bounded linear operators on $H$. By a projection we mean a self-adjoint idempotent in $B(H)$. For any $n \in \mathbb{N}, P_{n}(H)$ denotes the set of all rank- $n$ projections on $H$, and $P_{\infty}(H)$ stands for the set of all infinite rank projections. Clearly, $P_{n}(H)$ can be identified with the set of all $n$-dimensional subspaces of $H$. As it was mentioned in the abstract, Wigner's theorem describes the bijective trans- 
formations on the set $\mathcal{L}$ of all 1-dimensional subspaces of $H$ which preserve the angle between the elements of $\mathcal{L}$. It seems to be a very natural problem to try to extend this result from the 1-dimensional case to the case of higher dimensional subspaces (in our recent papers 11, [12, [13] we have presented several other generalizations of Wigner's theorem for different structures). But what about the angle between two higher dimensional subspaces of $H$ ? For our present purposes, the most adequate concept of angles is that of the so-called principal angles (or canonical angles, in a different terminology). This concept is a generalization of the usual notion of angles between 1-dimensional subspaces and reads as follows. If $P, Q$ are finite dimensional projections, then the principal angles between them (or, equivalently, between their ranges as subspaces) is defined as the arccos of the square root of the eigenvalues (counted according multiplicity) of the positive (self-adjoint) finite rank operator $Q P Q$ (see, for example, [1, Exercise VII.1.10] or [7, Problem 559]). We remark that this concept of angles was motivated by the classical work [3] of Jordan and it has serious applications in statistics, for example (see the canonical correlation theory of Hotelling [4], and also see the introduction of [9]). The system of all principal angles between $P$ and $Q$ is denoted by $\angle(P, Q)$. Thus, we have the desired concept of angles between finite rank projections. But in what follows we would like to extend Wigner's theorem also for the case of infinite rank projections. Therefore, we need the concept of principal angles also between infinite rank projections. Using deep concepts of operator theory (like scalar-valued spectral measure and multiplicity function) this could be carried out, but in order to formulate a Wigner-type result we need only the equality of angles. Hence, we can avoid these complications saying that for arbitrary projections $P, Q, P^{\prime}, Q^{\prime}$ on $H$ we have $\angle(P, Q)=\angle\left(P^{\prime}, Q^{\prime}\right)$ if and only if the positive operators $Q P Q$ and $Q^{\prime} P^{\prime} Q^{\prime}$ are unitarily equivalent. This obviously generalizes the equality of principal angles between pairs of finite rank projection. 
Keeping in mind the formulation of Wigner's theorem given in the abstract, we are now in a position to formulate the main result of the paper which, we believe, also has physical interpretation.

Main Theorem. Let $n \in \mathbb{N}$. Let $H$ be a real or complex Hilbert space with $\operatorname{dim} H \geq n$. Suppose that $\phi: P_{n}(H) \rightarrow P_{n}(H)$ is a transformation with the property that

$$
\angle(\phi(P), \phi(Q))=\angle(P, Q) \quad\left(P, Q \in P_{n}(H)\right) .
$$

If $n=1$ or $n \neq \operatorname{dim} H / 2$, then there exists a linear or conjugate-linear isometry $V$ on $H$ such that

$$
\phi(P)=V P V^{*} \quad\left(P \in P_{n}(H)\right)
$$

If $H$ is infinite dimensional, the transformation $\phi: P_{\infty}(H) \rightarrow P_{\infty}(H)$ satisfies

$$
\angle(\phi(P), \phi(Q))=\angle(P, Q) \quad\left(P, Q \in P_{\infty}(H)\right)
$$

and $\phi$ is surjective, then there exists a unitary or antiunitary operator $U$ on $H$ such that

$$
\phi(P)=U P U^{*} \quad\left(P \in P_{\infty}(H)\right)
$$

As one can suspect from the formulation of our main result, there is a system of exceptional cases, namely, when we have $\operatorname{dim} H=2 n, n>1$. In the next section we show that in those cases there do exist transformations on $P_{n}(H)$ which preserve the principal angles but cannot be written in the form appearing in our main theorem above.

\section{Proof}

This section is devoted to the proof of our main theorem. In fact, this will follow from the statements below.

The idea of the proof can be summarized in a single sentence as follows. We extend our transformation from $P_{n}(H)$ to a Jordan homomorphism of the algebra 
$F(H)$ of all finite rank operators on $H$ which preserves the rank-1 operators. Fortunately, those maps turn to have a form and using this we can achieve the desired conclusion. On the other hand, quite unfortunately, we have to work hard to carry out all the details of the proof that we are just going to begin.

From now on, let $H$ be a real or complex Hilbert space and let $n \in \mathbb{N}$. Since our statement obviously holds when $\operatorname{dim} H=n$, hence we suppose that $\operatorname{dim} H>n$.

In the sequel, let tr be the usual trace functional on operators. The ideal of all finite rank operators in $B(H)$ is denoted by $F(H)$. Clearly, every element of $F(H)$ has a finite trace. We denote by $F_{s}(H)$ the set of all self-adjoint elements of $F(H)$.

We begin with two key lemmas. In order to understand why we consider the property (1) in Lemma 且, we note that if $\angle(P, Q)=\angle\left(P^{\prime}, Q^{\prime}\right)$ for some finite rank projections $P, Q, P^{\prime}, Q^{\prime}$, then, by definition, the positive operators $Q P Q$ and $Q^{\prime} P^{\prime} Q^{\prime}$ are unitarily equivalent. This implies that $\operatorname{tr} Q P Q=\operatorname{tr} Q^{\prime} P^{\prime} Q^{\prime}$. But, by the properties of the trace, we have $\operatorname{tr} Q P Q=\operatorname{tr} P Q Q=\operatorname{tr} P Q$ and, similarly, $\operatorname{tr} Q^{\prime} P^{\prime} Q^{\prime}=\operatorname{tr} P^{\prime} Q^{\prime}$. So, if our transformation preserves the principal angles between projections, then it necessarily preserves the trace of the product of the projections in question. This justifies the condition (11) in the next lemma.

Lemma 1. Let $\mathcal{P}$ be any set of finite rank projections on $H$. If $\phi: \mathcal{P} \rightarrow \mathcal{P}$ is a transformation with the property that

$$
\operatorname{tr} \phi(P) \phi(Q)=\operatorname{tr} P Q \quad(P, Q \in \mathcal{P})
$$

then $\phi$ has a unique real-linear extension $\Phi$ onto the real-linear span $\operatorname{span}_{\mathbb{R}} \mathcal{P}$ of $\mathcal{P}$. The transformation $\Phi$ is injective, preserves the trace and satisfies

$$
\operatorname{tr} \Phi(A) \Phi(B)=\operatorname{tr} A B \quad\left(A, B \in \operatorname{span}_{\mathbb{R}} \mathcal{P}\right) .
$$

Proof. For any finite sets $\left\{\lambda_{i}\right\} \subset \mathbb{R}$ and $\left\{P_{i}\right\} \subset \mathcal{P}$ we define

$$
\Phi\left(\sum_{i} \lambda_{i} P_{i}\right)=\sum_{i} \lambda_{i} \phi\left(P_{i}\right)
$$


We have to show that $\Phi$ is well-defined. If $\sum_{i} \lambda_{i} P_{i}=\sum_{k} \mu_{k} Q_{k}$, where $\left\{\mu_{k}\right\} \subset \mathbb{R}$ and $\left\{Q_{k}\right\} \subset \mathcal{P}$ are finite subsets, then for any $R \in \mathcal{P}$ we compute

$$
\begin{gathered}
\operatorname{tr}\left(\sum_{i} \lambda_{i} \phi\left(P_{i}\right) \phi(R)\right)=\sum_{i} \lambda_{i} \operatorname{tr}\left(\phi\left(P_{i}\right) \phi(R)\right)=\sum_{i} \lambda_{i} \operatorname{tr}\left(P_{i} R\right)= \\
\operatorname{tr}\left(\sum_{i} \lambda_{i} P_{i} R\right)=\operatorname{tr}\left(\sum_{k} \mu_{k} Q_{k} R\right)=\sum_{k} \mu_{k} \operatorname{tr}\left(Q_{k} R\right)= \\
\sum_{k} \mu_{k} \operatorname{tr}\left(\phi\left(Q_{k}\right) \phi(R)\right)=\operatorname{tr}\left(\sum_{k} \mu_{k} \phi\left(Q_{k}\right) \phi(R)\right) .
\end{gathered}
$$

Therefore, we have

$$
\operatorname{tr}\left(\left(\sum_{i} \lambda_{i} \phi\left(P_{i}\right)-\sum_{k} \mu_{k} \phi\left(Q_{k}\right)\right) \phi(R)\right)=0
$$

for every $R \in \mathcal{P}$. By the linearity of the trace functional it follows that we have similar equality if we replace $\phi(R)$ by any finite linear combination of $\phi(R)$ 's. This gives us that

$$
\operatorname{tr}\left(\left(\sum_{i} \lambda_{i} \phi\left(P_{i}\right)-\sum_{k} \mu_{k} \phi\left(Q_{k}\right)\right)\left(\sum_{i} \lambda_{i} \phi\left(P_{i}\right)-\sum_{k} \mu_{k} \phi\left(Q_{k}\right)\right)\right)=0
$$

The operator $\left(\sum_{i} \lambda_{i} \phi\left(P_{i}\right)-\sum_{k} \mu_{k} \phi\left(Q_{k}\right)\right)^{2}$, being the square of a self-adjoint operator, is positive. Since its trace is zero, we obtain that

$$
\left(\sum_{i} \lambda_{i} \phi\left(P_{i}\right)-\sum_{k} \mu_{k} \phi\left(Q_{k}\right)\right)^{2}=0
$$

which plainly implies that

$$
\sum_{i} \lambda_{i} \phi\left(P_{i}\right)-\sum_{k} \mu_{k} \phi\left(Q_{k}\right)=0
$$

This shows that $\Phi$ is well-defined. The real-linearity of $\Phi$ now follows from the definition. The uniqueness of $\Phi$ is also trivial to see. From (1) we immediately obtain (2). One can introduce an inner product on $F_{s}(H)$ by the formula

$$
\langle A, B\rangle=\operatorname{tr} A B \quad\left(A, B \in F_{s}(H)\right)
$$


(the norm induced by this inner product is called the Hilbert-Schmidt norm). The equality (2) shows that $\Phi$ is an isometry with respect to this norm. Thus, $\Phi$ is injective. It follows from (1) that

$$
\operatorname{tr} \phi(P)=\operatorname{tr} \phi(P)^{2}=\operatorname{tr} P^{2}=\operatorname{tr} P \quad(P \in \mathcal{P})
$$

which clearly implies that

$$
\operatorname{tr} \Phi(A)=\operatorname{tr} A \quad\left(A \in \operatorname{span}_{\mathbb{R}} \mathcal{P}\right) .
$$

This completes the proof of the lemma.

In what follows we need the concept of Jordan homomorphisms. If $\mathcal{A}$ and $\mathcal{B}$ are algebras, then a linear transformation $\Psi: \mathcal{A} \rightarrow \mathcal{B}$ is called a Jordan homomorphism if it satisfies

$$
\Psi\left(A^{2}\right)=\Psi(A)^{2} \quad(A \in \mathcal{A}),
$$

or, equivalently, if

$$
\Psi(A B+B A)=\Psi(A) \Psi(B)+\Psi(B) \Psi(A) \quad(A, B \in \mathcal{A})
$$

Two projections $P, Q$ on $H$ are said to be orthogonal if $P Q=Q P=0$ (this means that the ranges of $P$ and $Q$ are orthogonal to each other). In this case we write $P \perp Q$. We denote $P \leq Q$ if $P Q=Q P=P$ (this means that the range of $P$ is included in the range of $Q$ ). In what follows, we shall use the following useful notation. If $x, y \in H$, then $x \otimes y$ stands for the operator defined by

$$
(x \otimes y) z=\langle z, y\rangle x \quad(z \in H) .
$$

Lemma 2. Let $\Phi: F_{s}(H) \rightarrow F_{s}(H)$ be a real-linear transformation which preserves the rank-1 projections and the orthogonality between them. Then there is an either linear or conjugate-linear isometry $V$ on $H$ such that

$$
\Phi(A)=V A V^{*} \quad\left(A \in F_{s}(H)\right)
$$


Proof. Since every finite-rank projection is the finite sum of pairwise orthogonal rank-1 projections, it is obvious that $\Phi$ preserves the finite-rank projections. It follows from [2, Remark 2.2] and the spectral theorem that $\Phi$ is a Jordan homomorphism (we note that [2, Remark 2.2] is about self-adjoint operators on finite dimensional complex Hilbert spaces, but the same argument applies for $F_{s}(H)$ even if it is infinite dimensional and/or real).

We next prove that $\Phi$ can be extended to a Jordan homomorphism of $F(H)$. To see this, first suppose that $H$ is complex and consider the transformation $\tilde{\Phi}: F(H) \rightarrow F(H)$ defined by

$$
\tilde{\Phi}(A+i B)=\Phi(A)+i \Phi(B) \quad\left(A, B \in F_{s}(H)\right) .
$$

It is easy to see that $\Phi$ is a linear transformation which satisfies $\tilde{\Phi}\left(T^{2}\right)=\tilde{\Phi}(T)^{2}$ $(T \in F(H))$. This shows that $\tilde{\Phi}$ is a Jordan homomorphism.

If $H$ is real, then the situation is not so simple, but we can apply a deep algebraic result of Martindale as follows (cf. the proof of [10, Theorem 3]). Consider the unitalized algebra $F(H) \oplus \mathbb{R} I$ (of course, we have to add the identity only when $H$ is infinite dimensional). Defining $\Phi(I)=I$, we can extend $\Phi$ to the set of all symmetric elements of the enlarged algebra in an obvious way. Now we are in a position to apply the results in [8] on the extendability of Jordan homomorphisms defined on the set of symmetric elements of a ring with involution. To be precise, in [8] Jordan homomorphism means an additive map $\Psi$ which, besides $\Psi\left(s^{2}\right)=\Psi(s)^{2}$, also satisfies $\Psi(s t s)=\Psi(s) \Psi(t) \Psi(s)$. But if the ring in question is 2-torsion free (in particular, if it is an algebra), this second equality follows from the first one (see, for example, the proof of [15, 6.3.2 Lemma]). The statements [8, Theorem 1] in the case when $\operatorname{dim} H \geq 3$ and [8, Theorem 2] if $\operatorname{dim} H=2$ imply that $\Phi$ can be uniquely extended to an associative homomorphism of $F(H) \oplus \mathbb{R} I$ into itself. To be honest, since the results of Martindale concern rings and hence linearity does not appear, we could guarantee only the additivity of the extension of $\Phi$. However, the construction in [8] shows that in the case of algebras, linear Jordan homomorphisms have linear extensions. 
To sum up, in every case we have a Jordan homomorphism of $F(H)$ extending $\Phi$. In order to simplify the notation, we use the same symbol $\Phi$ for the extension as well.

As $F(H)$ is a locally matrix ring (every finite subset of $F(H)$ can be included in a subalgebra of $F(H)$ which is isomorphic to a full matrix algebra), it follows from a classical result of Jacobson and Rickart [6, Theorem 8] that $\Phi$ can be written as $\Phi=\Phi_{1}+\Phi_{2}$, where $\Phi_{1}$ is a homomorphism and $\Phi_{2}$ is an antihomomorphism. Let $P$ be a rank-1 projection on $H$. Since $\Phi(P)$ is also rank-1, we obtain that one of the idempotents $\Phi_{1}(P), \Phi_{2}(P)$ is zero. Since $F(H)$ is a simple ring, it is easy to see that this implies that either $\Phi_{1}$ or $\Phi_{2}$ is identically zero, that is, $\Phi$ is either a homomorphism or an antihomomorphism of $F(H)$. In what follows we can assume without loss of generality that $\Phi$ is a homomorphism. Since the kernel of $\Phi$ is an ideal in $F(H)$ and $F(H)$ is simple, we obtain that $\Phi$ is injective.

We show that $\Phi$ preserves the rank-1 operators. Let $A \in F(H)$ be of rank 1. Then there is a rank-1 projection $P$ such that $P A=A$. We have $\Phi(A)=$ $\Phi(P A)=\Phi(P) \Phi(A)$ which proves that $\Phi(A)$ is of rank at most 1 . Since $\Phi$ is injective, we obtain that the rank of $\Phi(A)$ is exactly 1 . From the conditions of the lemma it follows that $\phi$ sends rank-2 projections to rank-2 projections. Therefore, the range of $\Phi$ contains an operator with rank greater than 1 . We now refer to Hou's work [5] on the form of linear rank preservers on operator algebras. It follows from the argument leading to [0, Theorem 1.3] that either there are linear operators $T, S$ on $H$ such that $\Phi$ is of the form

$$
\Phi(x \otimes y)=(T x) \otimes(S y) \quad(x, y \in H)
$$

or there are conjugate-linear operators $T^{\prime}, S^{\prime}$ on $H$ such that $\Phi$ is of the form

$$
\Phi(x \otimes y)=\left(S^{\prime} y\right) \otimes\left(T^{\prime} x\right) \quad(x, y \in H) .
$$


Suppose that we have the first possibility. By the multiplicativity of $\Phi$ we obtain that

$$
\begin{gathered}
\langle u, y\rangle T x \otimes S v=\langle u, y\rangle \Phi(x \otimes v)=\Phi(x \otimes y \cdot u \otimes v)= \\
\Phi(x \otimes y) \Phi(u \otimes v)=\langle T u, S y\rangle T x \otimes S v .
\end{gathered}
$$

This gives us that $\langle T u, S y\rangle=\langle u, y\rangle$ for every $u, y \in H$. On the other hand, since $\Phi$ sends rank-1 projections to rank-1 projections, we obtain that for every unit vector $x \in H$ we have $T x=S x$. These imply that $T=S$ is an isometry and with the notation $V=T=S$ we have

$$
\Phi(A)=V A V^{*}
$$

for every $A \in F_{s}(H)$.

We show that the possibility (3) cannot occur. In fact, similarly to (4) we have

$$
\langle u, y\rangle S^{\prime} v \otimes T^{\prime} x=\left\langle S^{\prime} v, T^{\prime} x\right\rangle S^{\prime} y \otimes T^{\prime} u \quad(x, y, u, v \in H)
$$

Fixing unit vectors $x=y=u$ in $H$ and considering the operators above at $T^{\prime} x$, we find that

$$
S^{\prime} v=\left\langle S^{\prime} v, T^{\prime} x\right\rangle\left\langle T^{\prime} x, T^{\prime} u\right\rangle S^{\prime} y
$$

giving us that $S^{\prime}$ is of rank 1 . Since $\Phi$ sends rank-2 projections to rank-2 projections, we arrive at a contradiction. This completes the proof of the lemma.

We are now in a position to present a new proof of the nonsurjective version of Wigner's theorem which is equivalent to the statement of our main theorem in the case when $n=1$. For another proof see [16].

To begin, observe that if $P, Q$ are finite rank projections such that $\operatorname{tr} P Q=0$, then we have $\operatorname{tr}(P Q)^{*} P Q=\operatorname{tr} Q P Q=\operatorname{tr} P Q Q=\operatorname{tr} P Q=0$ which implies that $(P Q)^{*}(P Q)=0$. This gives us that $P Q=0=Q P$. Therefore, $P$ is orthogonal to $Q$ if and only if $\operatorname{tr} P Q=0$. 
Theorem 3. Let $\phi: P_{1}(H) \rightarrow P_{1}(H)$ be a transformation with the property that

$$
\operatorname{tr} \phi(P) \phi(Q)=\operatorname{tr} P Q \quad\left(P, Q \in P_{1}(H)\right)
$$

Then there is an either linear or conjugate-linear isometry $V$ on $H$ such that

$$
\phi(P)=V P V^{*} \quad\left(P \in P_{1}(H)\right) .
$$

Proof. By the spectral theorem it is obvious that the real linear span of $P_{1}(H)$ is $F_{s}(H)$. Then, by Lemma 1 we see that there is a unique real-linear extension $\Phi$ of $\phi$ onto $F_{s}(H)$ which preserves the rank-1 projections and, by (5), $\Phi$ also preserves the orthogonality between the elements of $P_{1}(H)$. Lemma 2 applies to complete the proof.

As for the cases when $n>1$ we need the following lemma. Recall that we have previously supposed that $\operatorname{dim} H>n$.

Lemma 4. Let $1<n \in \mathbb{N}$. Then $\operatorname{span}_{\mathbb{R}} P_{n}(H)$ coincides with $F_{s}(H)$.

Proof. Since the real-linear span of $P_{1}(H)$ is $F_{s}(H)$, it is sufficient to show that every rank-1 projection is a real-linear combination of rank- $n$ projections. To see this, choose orthonormal vectors $e_{1}, \ldots, e_{n+1}$ in $H$. Let $E=e_{1} \otimes e_{1}+\ldots+$ $e_{n+1} \otimes e_{n+1}$ and define

$$
P_{k}=E-e_{k} \otimes e_{k} \quad(k=1, \ldots, n+1) .
$$

Clearly, every $P_{k}$ can be represented by a $(n+1) \times(n+1)$ diagonal matrix whose diagonal entries are all 1's with the exception of the $k^{\text {th }}$ one which is 0 . The equation

$$
\lambda_{1} P_{1}+\ldots+\lambda_{n+1} P_{n+1}=e_{1} \otimes e_{1}
$$

gives rise to a system of linear equations with unknown scalars $\lambda_{1}, \ldots, \lambda_{n+1}$. The matrix of this system of equations is an $(n+1) \times(n+1)$ matrix whose diagonal consists of 0 's and its off-diagonal entries are all 1 's. It is easy to see that this matrix is nonsingular, and hence $e_{1} \otimes e_{1}$ (and, similarly, every other 
$\left.e_{k} \otimes e_{k}\right)$ is a real-linear combination of $P_{1}, \ldots, P_{n+1}$. This completes the proof.

We continue with a technical lemma.

Lemma 5. Let $P, Q$ be projections on $H$. If $Q P Q$ is a projection, then there are pairwise orthogonal projections $R, R^{\prime}, R^{\prime \prime}$ such that $P=R+R^{\prime}, Q=R+R^{\prime \prime}$. In particular, we obtain that $Q P Q$ is a projection if and only if $P Q=Q P$.

Proof. Let $R=Q P Q$. Since $R$ is a projection whose range is contained in the range of $Q$, it follows that $R^{\prime \prime}=Q-R$ is a projection which is orthogonal to $R$.

If $x$ is a unit vector in the range of $R$, then we have $\|Q P Q x\|=1$. Since $P Q x$ is a vector whose norm is at most 1 and its image under the projection $Q$ has norm 1, we obtain that $P Q x$ is a unit vector in the range of $Q$. Similarly, we obtain that $Q x$ is a unit vector in the range of $P$ and, finally, that $x$ is a unit vector in the range of $Q$. Therefore, $x$ belongs to the range of $P$ and $Q$. Since $x$ was arbitrary, we can infer that the range of $R$ is included in the range of $P$. Thus, we obtain that $R^{\prime}=P-R$ is a projection which is orthogonal to $R$.

Next, using the obvious relations

$$
P R=R P=R, \quad Q R=R Q=R
$$

we deduce

$$
\begin{gathered}
(Q-R)(P-R)(Q-R)= \\
Q P Q-Q P R-Q R Q+Q R-R P Q+R P R+R Q-R= \\
R-R-R+R-R+R+R-R=0 .
\end{gathered}
$$

Since $A^{*} A=0$ implies $A=0$ for any $A \in B(H)$, we obtain from (6) that $R^{\prime} R^{\prime \prime}=(P-R)(Q-R)=0$.

The second part of the assertion is now easy to check.

We next prove the assertion of our main theorem in the case when $1<n \in \mathbb{N}$ and $H$ is infinite dimensional. 
Theorem 6. Suppose $1<n \in \mathbb{N}$ and $H$ is infinite dimensional. If $\phi: P_{n}(H) \rightarrow$ $P_{n}(H)$ is a transformation such that

$$
\angle(\phi(P), \phi(Q))=\angle(P, Q) \quad\left(P, Q \in P_{n}(H)\right),
$$

then there exists a linear or conjugate-linear isometry $V$ on $H$ such that

$$
\phi(P)=V P V^{*} \quad\left(P \in P_{n}(H)\right) .
$$

Proof. By Lemma 1 and Lemma 4 , $\phi$ can be uniquely extended to an injective real-linear transformation $\Phi$ on $F_{s}(H)$. The main point of the proof is to show that $\Phi$ preserves the rank-1 projections. In order to verify this, just as in the proof of Lemma 4, we consider orthonormal vectors $e_{1}, \ldots, e_{n+1}$ in $H$, define $E=e_{1} \otimes e_{1}+\ldots+e_{n+1} \otimes e_{n+1}$ and set

$$
P_{k}=E-e_{k} \otimes e_{k} \quad(k=1, \ldots, n+1) .
$$

We show that the ranges of all $P_{k}^{\prime}=\phi\left(P_{k}\right)$ 's can be jointly included in an $(n+1)$-dimensional subspace of $H$. To see this, we first recall that $\Phi$ has the property that

$$
\operatorname{tr} \Phi(A) \Phi(B)=\operatorname{tr} A B \quad\left(A, B \in F_{s}(H)\right)
$$

(see Lemma 1 ). Next we have the following property of $\Phi$ : if $P, Q$ are orthogonal rank-1 projections, then $\Phi(P) \Phi(Q)=0$. Indeed, if $P, Q$ are orthogonal, then we can include them into two orthogonal rank- $(n+1)$ projections. Now, referring to the construction given in Lemma 1 and having in mind that $\Phi$ preserves the orthogonality between rank- $n$ projections, we obtain that $\Phi(P) \Phi(Q)=0$. (Clearly, the same argument works if $\operatorname{dim} H \geq 2(n+1)$.) Since the rank- $n$ projections $P_{k}$ are commuting, by the preserving property of $\phi$ and Lemma 5, it follows that the projections $\Phi\left(P_{k}\right)$ are also commuting. It is well-known that any finite commuting family of operators in $F_{s}(H)$ can be diagonalized by the same unitary transformation (or, in the real case, by the same orthogonal transformation). Therefore, if we resctrict $\Phi$ onto the real-linear subspace in $F_{s}(H)$ generated by 
$P_{1}, \ldots, P_{n+1}$, then it can be identified with a real-linear operator from $\mathbb{R}^{n+1}$ to $\mathbb{R}^{m}$ for some $m \in \mathbb{N}$. Clearly, this restriction of $\Phi$ can be represented by an $m \times(n+1)$ real matrix $T=\left(t_{i j}\right)$. Let us examine how the properties of $\Phi$ are reflected in those of the matrix $T$. First, $\Phi$ is trace preserving. This gives us that for every $\underline{\lambda} \in \mathbb{R}^{n+1}$ the sums of the coordinates of the vectors $T \underline{\lambda}$ and $\underline{\lambda}$ are the same. This easily implies that the sum of the entries of $T$ lying in a fixed column is always 1 . As we have already noted, $\Phi\left(e_{i} \otimes e_{i}\right) \Phi\left(e_{j} \otimes e_{j}\right)=0$ holds for every $i \neq j$. For the matrix $T$ this means that the coordinatewise product of any two columns of $T$ is zero. Consequently in every row of $T$ there is at most one nonzero entry. Since $\Phi$ sends rank- $n$ projections to rank- $n$ projections, we see that this possibly nonzero entry is necessarily 1 . So, every row contains at most one 1 and all the other entries in that row are 0's. Since the sum of the elements in every column is 1 , we have that in every column there is exactly one 1 and all the other entries are 0's in that column. These now easily imply that if $\underline{\lambda} \in \mathbb{R}^{n+1}$ is such that its coordinates are all 0's with the exception of one which is 1 , then $T \underline{\lambda}$ is of the same kind. What concerns $\Phi$, this means that $\Phi$ sends every $e_{k} \otimes e_{k}(k=1, \ldots, n+1)$ to a rank-1 projection.

So, we obtain that $\Phi$ preserves the rank-1 projections and the orthogonality between them. Now, by Lemma 2 we conclude the proof.

We turn to the case when $H$ is finite dimensional.

Theorem 7. Suppose $1<n \in \mathbb{N}, H$ is finite dimensional and $n \neq \operatorname{dim} H / 2$. If $\phi: P_{n}(H) \rightarrow P_{n}(H)$ satisfies

$$
\angle(\phi(P), \phi(Q))=\angle(P, Q) \quad\left(P, Q \in P_{n}(H)\right),
$$

then there exists a unitary or antiunitary operator $U$ on $H$ such that

$$
\phi(P)=U P U^{*} \quad\left(P \in P_{\infty}(H)\right) .
$$

Proof. First suppose that $\operatorname{dim} H=2 d, 1<d \in \mathbb{N}$. If $n=1, \ldots, d-1$, then we can apply the method followed in the proof of Theorem 6 concerning the infinite 
dimensional case. If $n=d+1, \ldots, 2 d-1$, then consider the transformation $\psi: P \mapsto I-\phi(I-P)$ on $P_{2 d-n}(H)$. We learn from [7, Problem 559] that if $\angle(P, Q)=\angle\left(P^{\prime}, Q^{\prime}\right)$, then there exists a unitary operator $U$ such that $U P U^{*}=$ $P^{\prime}$ and $U Q U^{*}=Q^{\prime}$. It follows from the preserving property of $\phi$ that for any $P, Q \in P_{2 d-n}(H)$ we have

$$
\phi(I-P)=U(I-P) U^{*}, \quad \phi(I-Q)=U(I-Q) U^{*}
$$

for some unitary operator $U$ on $H$. This gives us that

$$
\angle(\psi(P), \psi(Q))=\angle\left(U P U^{*}, U Q U^{*}\right)=\angle(P, Q) .
$$

In that way we can reduce the problem to the previous case. So, there is an either unitary or antiunitary operator $U$ on $H$ such that

$$
\psi(P)=U P U^{*} \quad\left(P \in P_{2 d-n}(H)\right) .
$$

It follows that $\phi(I-P)=I-\psi(P)=I-U P U^{*}=U(I-P) U^{*}$, and hence we have the result for the considered case.

Next suppose that $\operatorname{dim} H=2 d+1, d \in \mathbb{N}$. If $n=1, \ldots, d-1$, then once again we can apply the method followed in the proof of Theorem 6. If $n=$ $d+2, \ldots, 2 d+1$, then using the 'dual method' that we have applied right above we can reduce the problem to the previous case. If $n=d$, consider a fixed rank- $d$ projection $P_{0}$. Clearly, if $P$ is any rank- $d$ projection orthogonal to $P_{0}$, then the rank- $d$ projection $\phi(P)$ is orthogonal to $\phi\left(P_{0}\right)$. Therefore, $\phi$ induces a transformation $\phi_{0}$ between $d+1$-dimensional spaces (namely, between the orthogonal complement of the range of $P_{0}$ and that of the range of $\left.\phi\left(P_{0}\right)\right)$ which preserves the principal angles between the rank- $d$ projections. Our 'dual method' and the result concerning 1-dimensional subspaces lead us to the conslusion that the linear extension of $\phi_{0}$ maps rank-1 projections to rank-1 projections and preserves the orthogonality between them. This implies that the same holds true for our original transformation $\phi$. Just as before, using Lemma 1 and Lemma 2 we can conclude the proof. In the remaining case $n=d+1$ we apply the 'dual method' once again. 
We now show that the case when $1<n \in \mathbb{N}, n=\operatorname{dim} H / 2$ is really exceptional. To see this, consider the transformation $\phi: P \mapsto I-P$ on $P_{n}(H)$. This maps $P_{n}(H)$ into itself and preserves the principal angles. As for the complex case, the preserving property follows from [1, Exercise VII.1.11] while in the real case it was proved already by Jordan in [3] (see [14], p. 310). Let us now suppose that the transformation $\phi$ can be written in the form (7). Pick a rank-1 projection $Q$ on $H$. We know that it is a real linear combination of some $P_{1}, \ldots, P_{n+1} \in P_{n}(H)$. It would follow from (7) that considering the same linear combination of $\phi\left(P_{1}\right), \ldots, \phi\left(P_{n+1}\right)$, it is a rank-1 projection as well. But due to the definition of $\phi$, we get that this linear combination is a constant minus $Q$. By the trace preserving property we obtain that this constant is $1 / n$. Since $n>1$, the operator $(1 / n) I-Q$ is obviously not a projection. Therefore, we have arrived at a contradiction. This shows that the transformation above can not be written in the form (7).

It would be a nice result if one could prove that in the present case (i.e., when $1<n, n=\operatorname{dim} H / 2)$ up to unitary-antiunitary equivalence, there are exactly two transformations on $P_{n}(H)$ preserving principal angles, namely, $P \mapsto P$ and $P \mapsto I-P$. This is left as an open problem.

We now turn to our statement concerning infinite rank projections. In the proof we shall use the following simple lemma. If $A \in B(H)$, then denote by $\operatorname{rng} A$ the range of $A$.

Lemma 8. Let $H$ be an infinite dimensional Hilbert space. Suppose $P, Q$ are projections on $H$ with the property that for any projection $R$ with finite corank we have $R P=P R$ if and only if $R Q=Q R$. Then either $P=Q$ or $P=I-Q$.

Proof. Let $R$ be any projection on $H$ commuting with $P$. By Lemma 5 , it is easy to see that we can choose a monotone decreasing net $\left(R_{\alpha}\right)$ of projections with finite corank such that $\left(R_{\alpha}\right)$ converges weakly to $R$ and $R_{\alpha}$ commutes with $P$ for every $\alpha$. Since $R_{\alpha}$ commutes with $Q$ for every $\alpha$, we obtain that 
$R$ commutes with $Q$. Interchanging the role of $P$ and $Q$, we obtain that any projection commutes with $P$ if and only if it commutes with $Q$.

Let $x$ be any unit vector from the range of $P$. Consider $R=x \otimes x$. Since $R$ commutes with $P$, it must commute with $Q$ as well. By Lemma f we obtain that $x$ belongs either to the range of $Q$ or to its orthogonal complement. It follows that either $d(x, \operatorname{rng} Q)=0$, or $d(x, \operatorname{rng} Q)=1$. Since the set of all unit vectors in the range of $P$ is connected and the distance function is continuous, we get that either every unit vector in $\operatorname{rng} P$ belongs to $\operatorname{rng} Q$ or every unit vector in $\operatorname{rng} P$ belongs to $(\operatorname{rng} Q)^{\perp}$. Interchanging the role of $P$ and $Q$, we find that either $\operatorname{rng} P=\operatorname{rng} Q$ or $\operatorname{rng} P=(\operatorname{rng} Q)^{\perp}$. This gives us that either $P=Q$ or $P=I-Q$.

Theorem 9. Let $H$ be an infinite dimensional Hilbert space. Suppose that $\phi$ : $P_{\infty}(H) \rightarrow P_{\infty}(H)$ is a surjective transformation with the property that

$$
\angle(\phi(P), \phi(Q))=\angle(P, Q) \quad\left(P, Q \in P_{\infty}(H)\right) .
$$

Then there exists a unitary or antiunitary operator $U$ on $H$ such that

$$
\phi(P)=U P U^{*} \quad\left(P \in P_{\infty}(H)\right) .
$$

Proof. We first prove that $\phi$ is injective. If $P, P^{\prime} \in P_{\infty}(H)$ and $\phi(P)=\phi\left(P^{\prime}\right)$, then by the preserving property of $\phi$ we have

$$
\angle(P, Q)=\angle\left(P^{\prime}, Q\right) \quad\left(Q \in P_{\infty}(H)\right) .
$$

Putting $Q=I$, we see that $P$ is unitarily equivalent to $P^{\prime}$. We distiguish two cases. First, let $P$ be of infinite corank. By (8), we deduce that for every $Q \in$ $P_{\infty}(H)$ we have $Q \perp P$ if and only if $Q \perp P^{\prime}$. This gives us that $P=P^{\prime}$. As the second possibility, let $P$ be of finite corank. Then $P, P^{\prime}$ can be written in the form $P=I-P_{0}$ and $P^{\prime}=I-P_{0}^{\prime}$, where, by the equivalence of $P, P^{\prime}$, the projections $P_{0}$ and $P_{0}^{\prime}$ have finite and equal rank. Let $Q_{0}$ be any finite rank projection on $H$. It follows from

$$
\angle\left(I-P_{0}, I-Q_{0}\right)=\angle\left(I-P_{0}^{\prime}, I-Q_{0}\right)
$$


that there is a unitary operator $W$ on $H$ such that

$$
W\left(I-Q_{0}\right)\left(I-P_{0}\right)\left(I-Q_{0}\right) W^{*}=\left(I-Q_{0}\right)\left(I-P_{0}^{\prime}\right)\left(I-Q_{0}\right) .
$$

This implies that

$$
\begin{gathered}
W\left(-Q_{0}-P_{0}+P_{0} Q_{0}+Q_{0} P_{0}-Q_{0} P_{0} Q_{0}\right) W^{*}= \\
-Q_{0}-P_{0}^{\prime}+P_{0}^{\prime} Q_{0}+Q_{0} P_{0}^{\prime}-Q_{0} P_{0}^{\prime} Q_{0} .
\end{gathered}
$$

Taking traces, by the equality of the rank of $P_{0}$ and $P_{0}^{\prime}$, we obtain that

$$
\operatorname{tr} P_{0} Q_{0}=\operatorname{tr} P_{0}^{\prime} Q_{0}
$$

Since this holds for every finite rank projection $Q_{0}$ on $H$, it follows that $P_{0}=P_{0}^{\prime}$ and hence we have $P=P^{\prime}$. This proves the injectivity of $\phi$.

Let $P \in P_{\infty}(H)$ be of infinite corank. Then there is a projection $Q \in P_{\infty}(H)$ such that $Q \perp P$. By the preserving property of $\phi$, this implies that $\phi(Q) \perp \phi(P)$ which means that $\phi(P)$ is of infinite corank. One can similarly prove that if $\phi(P)$ is of infinite corank, then the same must hold for $P$. This yields that $P \in P_{\infty}(H)$ is of finite corank if and only if so is $\phi(P)$.

Denote by $P_{f}(H)$ the set of all finite rank projections on $H$. It follows that the transformation $\psi: P_{f}(H) \rightarrow P_{f}(H)$ defined by

$$
\psi(P)=I-\phi(I-P) \quad\left(P \in P_{f}(H)\right)
$$

is well-defined and bijective. Since $\phi(I-P)$ is unitarily equivalent to $I-P$ for every $P \in P_{f}(H)$ (this is because $\left.\angle(\phi(I-P), \phi(I-P))=\angle(I-P, I-P)\right)$, it follows that $\psi$ is rank preserving.

We next show that

$$
\operatorname{tr} \psi(P) \psi(Q)=\operatorname{tr} P Q \quad\left(P, Q \in P_{f}(H)\right) .
$$

This can be done following the argument leading to (9). In fact, by the preserving property of $\phi$ there is a unitary operator $W$ on $H$ such that

$$
W(I-\psi(Q))(I-\psi(P))(I-\psi(Q)) W^{*}=(I-Q)(I-P)(I-Q) .
$$


This gives us that

$$
\begin{gathered}
W(-\psi(Q)-\psi(P)+\psi(P) \psi(Q)+\psi(Q) \psi(P)-\psi(Q) \psi(P) \psi(Q)) W^{*}= \\
-Q-P+P Q+Q P-Q P Q .
\end{gathered}
$$

Taking traces on both sides and referring to the rank preserving property of $\psi$, we obtain (10). According to Lemma 1, let $\Psi: F_{s}(H) \rightarrow F_{s}(H)$ denote the unique real-linear extension of $\psi$ onto $\operatorname{span}_{\mathbb{R}} P_{f}(H)=F_{s}(H)$. We know that $\Psi$ is injective. Since $P_{f}(H)$ is in the range of $\Psi$, we obtain that $\Psi$ is surjective as well. It is easy to see that Lemma 2 can be applied and we infer that there exists an either unitary or antiunitary operator $U$ on $H$ such that

$$
\Psi(A)=U A U^{*} \quad\left(A \in F_{s}(H)\right)
$$

Therefore, we have

$$
\phi(P)=U P U^{*}
$$

for every projection $P \in P_{\infty}(H)$ with finite corank. It remains to prove that the same holds true for every $P \in P_{\infty}(H)$ with infinite corank as well. This could be quite easy to show if we know that $\phi$ preserves the order between the elements of $P_{\infty}(H)$. But this property is far away from being easy to verify. So we choose a different approach to attack the problem.

Let $P \in P_{\infty}(H)$ be a projection of infinite corank. By the preserving property of $\phi$ we see that for every $Q \in P_{\infty}(H)$ the operator $\phi(Q) \phi(P) \phi(Q)$ is a projection if and if $Q P Q$ is a projection. By Lemma 5 , this means that $\phi(Q)$ commutes with $\phi(P)$ if and only if $Q$ commutes with $P$. Therefore, for any $Q \in P_{\infty}(H)$ of finite corank, we obtain that $Q$ commutes with $U^{*} \phi(P) U$ (this is equivalent to that $\phi(Q)=U Q U^{*}$ commutes with $\left.\phi(P)\right)$ if and only if $Q$ commutes with $P$.

By Lemma 8 we have two possibilities, namely, either $U^{*} \phi(P) U=P$ or $U^{*} \phi(P) U=I-P$. Suppose that $U^{*} \phi(P) U=I-P$. Consider a complete orthonormal basis $e_{0}, e_{\gamma}(\gamma \in \Gamma)$ in the range of $P$ and, similarly, choose a 
complete orthonormal basis $f_{0}, f_{\delta}(\delta \in \Delta)$ in the range of $I-P$. Pick nonzero scalars $\lambda, \mu$ with the property that $|\lambda|^{2}+|\mu|^{2}=1$ and $|\lambda| \neq|\mu|$. Define

$$
Q=\left(\lambda e_{0}+\mu f_{0}\right) \otimes\left(\lambda e_{0}+\mu f_{0}\right)+\sum_{\gamma} e_{\gamma} \otimes e_{\gamma}+\sum_{\delta} f_{\delta} \otimes f_{\delta}
$$

Clearly, $Q$ is of finite corank (in fact, its corank is 1). Since $\phi(Q) \phi(P) \phi(Q)=$ $U Q U^{*} \phi(P) U Q U^{*}$ is unitarily equivalent to $Q P Q$, it follows that the spectrum of $Q U^{*} \phi(P) U Q$ is equal to the spectrum of $Q P Q$. This gives us that the spectrum of $Q(I-P) Q$ is equal to the spectrum of $Q P Q$. By the construction of $Q$ this means that

$$
\left\{0,1,|\mu|^{2}\right\}=\left\{0,1,|\lambda|^{2}\right\}
$$

which is an obvious contradiction. Consequently, we have $U^{*} \phi(P) U=P$, that is, $\phi(P)=U P U^{*}$. Thus, we have proved that this latter equality holds for every $P \in P_{\infty}(H)$ and the proof is complete.

Acknowledgements. This research was supported from the following sources: (1) Hungarian National Foundation for Scientific Research (OTKA), Grant No. T030082, T031995, (2) A grant from the Ministry of Education, Hungary, Reg. No. FKFP 0349/2000.

\section{References}

1. Bhatia, R.: Matrix Analysis. Springer-Verlag, 1997

2. Brešar, M., Šemrl, P.: Mappings which preserve idempotents, local automorpisms, and local derivations. Canad. J. Math. 45, 483-496 (1993)

3. Jordan, C.: Essai sur la géométrie á $n$ dimensions. Bull. Soc. Math. France 3, 103-174 (1875)

4. Hotelling, H.: Relations between two set of variates. Biometrika 28, 321-377 (1935)

5. Hou, J.C.: Rank-preserving linear maps on $B(X)$. Sci. China Ser. A 32, 929-940 (1989)

6. Jacobson, N., Rickart, C.: Jordan homomorphisms of rings. Trans. Amer. Math. Soc. 69, 479-502 (1950)

7. Kirillov, A.A., Gvishiani, A.D.: Theorems and Problems in Functional Analysis. Springer-Verlag, 1982

8. Martindale, W.S.: Jordan homomorphisms of the symmetric elements of a ring with involution. J. Algebra 5, 232-249 (1967) 
9. Miao, J., Ben-Israel, A.: On principal angles between subspaces in $\mathbb{R}^{n}$. Linear Algebra Appl. 171 81-98 (1992)

10. Molnár, L.: An algebraic approach to Wigner's unitary-antiunitary theorem. J. Austral. Math. Soc. 65, 354-369 (1998)

11. Molnár, L.: A generalization of Wigner's unitary-antiunitary theorem to Hilbert modules. J. Math. Phys. 40, 5544-5554 (1999)

12. Molnár, L.: Generalization of Wigner's unitary-antiunitary theorem for indefinite inner product spaces. Commun. Math. Phys. 201, 785-791 (2000)

13. Molnár, L.: A Wigner-type theorem on symmetry transformations in type II factors. Int. J. Theor. Phys. to appear

14. Paige, C.C., Wei, M.: History and generality of the CS decomposition. Linear Algebra Appl. 208/209, 303-326 (1994)

15. Palmer, T.W.: Banach Algebras and The General Theory of *-Algebras, Vol. I. Cambridge University Press, 1994

16. Sharma, C.S., Almeida, D.F.: A direct proof of Wigner's theorem on maps which preserve transition probabilities between pure states of quantum systems. Ann. Phys. 197, 300-309 (1990)

Communicated by H. Araki 